\title{
Unboxing mathematics: creating a culture of modeling as critic
}

\author{
Antonnette M. Gibbs ${ }^{1}$ (D) Joo Young Park ${ }^{2}$ (D)
}

Accepted: 8 October 2021 / Published online: 9 January 2022

(c) The Author(s), under exclusive licence to Springer Nature B.V. 2021

\begin{abstract}
From the socio-critical perspective of mathematical modeling, reflexive discussions about the nature, criteria, and consequences of mathematical models are not a natural consequence of modeling in school. This report is part of a larger study focused on stimulating reflexive discussions in practice employing constructivist grounded theory as a research method. Twenty-seven college algebra students engaged in a 3-week modeling project at a community college in the USA. Audio-recorded group discussions and written reflections were collected to determine how reflexive discussions were taking place. Analysis of students' actions and reflexive discussions during the modeling project produced four concepts: voicing mathematics, personalizing mathematics, challenging mathematics, and negotiating mathematics. These concepts are integrated into an overall process for stimulating reflexive discussions and are conceptualized as unboxing mathematics. The overarching concept of unboxing mathematics represents one interpretation of how reflexive discussions may be constituted during modeling activities and identifies classroom mathematical practices specific to the socio-critical modeling context of this study.
\end{abstract}

Keywords Socio-critical mathematical modeling $\cdot$ Reflexive discussions $\cdot$ Role of mathematics in society $\cdot$ Unboxing mathematics

\section{Introduction}

Applications of mathematical models in non-mathematical spheres of society exert consequential impact on society (Niss, 1994; Skovsmose, 1994, Seah \& Bishop, 2002). While society is being increasingly shaped by mathematics, citizens use less mathematics (Jablonka \& Gellert, 2007) which arguably poses detrimental consequences to citizenship and democracy (Bishop, 1999; D’Ambrosio, 1999; Niss, 1994; Skovsmose, 1994). In the light of the COVID-19 pandemic, the social, economic, and political discourse surrounding

Antonnette M. Gibbs

agibbs@broward.edu

Joo Young Park

jpark@fit.edu

1 Department of Mathematics, Broward College North Campus, Coconut Creek, FL, USA

2 Department of Mathematical Sciences, Florida Institute of Technology, Melbourne, FL, USA 
the crisis has shown us the urgent necessity and adequacy of a critical and reflective mathematics education (Bakker, Cai, \& Zenger, 2021; Kolosche \& Meyerhöfer, 2021; Skovsmose, 2021; Stephan et.al., 2021).

Historically, there has been a consistent call for mathematics education to develop critical, engaged, and productive citizens capable of deconstructing and reacting to the impact of mathematics in their everyday lives (Gutstein, 2006; National Council of Teachers of Mathematics (NCTM), 1989; National Governors Association Center for Best Practices, Council of Chief State School Officers (CCSSM), 2010; Niss, 1994; Organization for Economic Cooperation and Development (OECD), 2013; Pollak, 2007; Skovsmose, 1994). Perspectives and pedagogical practices that connect mathematics and mathematics education to the development of citizenship and democracy include the program ethnomathematics (D’Ambrosio, 1999), critical mathematics education (Bishop, 1988, 1990; Frankenstein, 1983; Skovsmose, 1994), mathematics for democracy (Niss, 1994), and mathematics for social justice (Atweh, 2007, Gutstein, 2003). These varying perspectives have coalesced into a resounding rejection of the universality, neutrality, and cultural-free nature of Western mathematics and prioritize critique and reflection on the role of mathematics in a democratic and just society.

There is no consensus on how mathematics education can accomplish such aspirations; however, mathematical modeling has proven essential in pedagogical practice and forms part of national curriculum documents and international assessments (CCSSM, 2010; NCTM, 1989; OECD, 2013). Mathematical modeling and applications in school usually focus on one of two goals: the use of models to learn mathematics content or develop modeling competencies (Blum et al., 2006; Julie, 2002). Barbosa (2006) argues for an explicit third goal of school modeling activities which prioritizes reflection upon the role of mathematics in society and is identified as socio-critical modeling. Socio-critical modeling is one of the six modeling perspectives outlined by Kaiser and Sriraman (2006) and is rooted to D’Ambrosio's (1999) program ethnomathematics and Skovsmose's $(1994,2011)$ critical mathematics education. The socio-critical perspective on mathematical modeling is distinguished by its focus on: a critical view of mathematical models and the consequences of these models in society, applying modeling to real-life problems (social, political, economic, etc.), empowerment of students, and development of reflexive discourse among students (Blomhøj, 2009; Kaiser \& Sriraman, 2006). Barbosa (2006) has used the metaphor, modeling as critic to describe the socio-critical perspective, which distinguishes it from the aforementioned foci of mathematical modeling in school and emphasizes the necessity of deliberate critique.

According to Barbosa (2006), socio-critical modeling is constituted in practice through student reflexive discussions during modeling. Reflexive discussion refers to discourse about the nature, criteria, and consequences of the social issue under investigation, the modeling process, the products of the modeling process (models), and the consequences of the application of those models in society (Barbosa, 2006; Skovsmose, 1994). Other types of discussions during modeling activities include mathematical and technological discussions (Barbosa, 2006). Mathematical discussion refers to discussions about mathematics concepts, calculations, formulas, etc. relevant to the task at hand, whereas technological discussions refer to discourse about constructing the model or completing the modeling task (Barbosa, 2006; Skovsmose, 1994).

Socio-critical modeling privileges the reflexive discussions (Barbosa 2006, 2010). While the transition between the technological, mathematical, and reflexive discussions occurs at points of stalemate or conflicts during the modeling process (Barbosa, 2006), there are two additional situations in which reflexive discussions may occur, comparative 
analysis of student-constructed models and debates about model criteria (Barbosa, 2009). However, the mere presence of reflexive discussions during the modeling activity is insufficient to develop a competence of critique, and "stimulating reflexive discussions should be a primary goal" (Barbosa, 2006, p. 298).

In practice, priority should be given to reflexive discussion with the other discussions as support and provide an opportunity for students to think about the role of mathematics in society (Barbosa, 2006). However, the modern view of mathematics does not lead to critical mathematics education priorities (Skovsmose, 2011). Little guidance can be found in the literature on how to design and plan modeling activities that would stimulate reflexive discussions in practice. The accessible literature on socio-critical modeling is due in part to the work of Barbosa $(2003,2006,2009,2010)$ who recommends further study into stimulating reflexive discussions in practice. Thus, this research was motivated by the sparse research on socio-critical mathematical modeling, the need to identify pedagogical practices for stimulating reflexive discussions and understanding the role of mathematics in society.

\subsection{A systematic approach to stimulating reflexive discussions}

Theoretically, it may be possible to stimulate reflexive discussions, but questions emerge regarding how modeling activities would look in practice. Blomhøj (2009) suggests that there is a need for further analysis of how to support the development of a student's critical consciousness during the modeling process. Skovsmose (1994) identifies three demands of critique: self-reflections, reflections, and reactions which form the basis of developing students' reflective competence. Self-reflection is the personal, individual reflection that may occur naturally in everyday life (Skovsmose, 1994). In the mathematical modeling context, engaging in self-reflections means to interrogate one's beliefs, values, criteria, and values relating to the social issue under investigation, the modeling process, productions of the modeling process, and consequences of these productions. Reflection utilizes the generally understood notion of what it means to engage in the process of "giving thought to actions" (Skovsmose, 2011, p. 71). However, in this study, attention is given to reflection with mathematics, reflection through mathematics, and reflection on mathematics (Skovsmose, 2011). Reaction is understood as the articulation of criticisms regarding the social situation resulting from self-reflections and reflections to make a change (Skovsmose \& Greer, 2012).

Galbraith (2012) presents a concise diagram of the steps of the modeling cycle which is reconstructed in Fig. 1 using gerunds to emphasize the active process of modeling. Arguably, steps one, two, three, and five are susceptibility to inherent biases; impact how the mathematical model is developed and used in decision-making; and should be subjected to scrutiny. During these steps, it may be possible to use direct critique and reflection activities to stimulate reflexive discussions. While it is important to critique the modeling process (cycle) itself, the productions of modeling and the consequences of such productions should also be subjected to critique.

A theoretical representation of the connection between mathematical modeling and the role of mathematics in society is presented in Fig. 2. The dark lines represent the connections that students need to make; however, it is not guaranteed that students can form these connections through the mathematical modeling process alone and modeling should be extended to include a critical element. 
$\downarrow 1$. Understanding the real problem situation

2. Framing an appropriate mathematical question

3. Formulating a model, using simplifying assumptions etc.

4. Analysing the model

5. Comparing mathematical outcomes with reality

6. Modifying and repeating until an adequate solution has been found

Fig. 1 Galbraith's (2012) modeling cycle diagram recreated using gerunds

Uniting Skovsmose's (2011) demands for critique, Blomhøj's (2009) three sources of critical reflection during mathematical modeling, and Barbosa's (2006) three types of discussions during modeling, we designed a tool called MESH (Mathematics Expressing Society's Hopes) as a systematic approach to stimulating reflexive discussions. The tool considers the steps in the modeling cycle (see Fig. 1) and prescribes reflections. The MESH tool provided a scaffold to help students connect mathematical modeling to the role of mathematics in society and consists of three cooperative activities and individual reflections after each activity (see Table 1). The stated goals of MESH are accomplished through processes of self-reflection, reflection, and reaction while engaging in the three activities sequential and working in cooperative groups. See Gibbs (2019) for an extensive discussion of the critical features of the MESH tool.

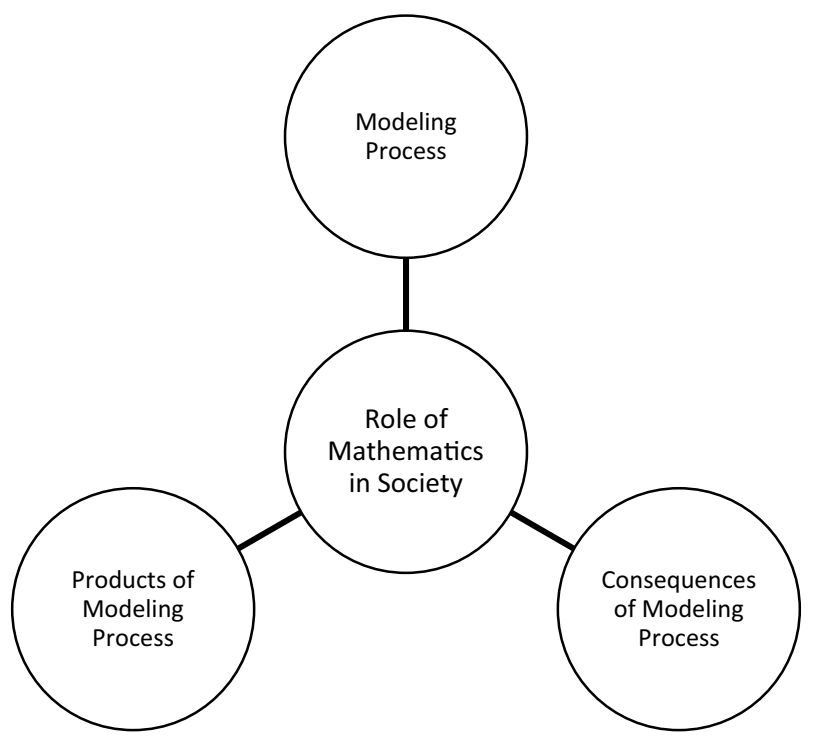

Fig. 2 Factors that influence the process of understanding the role of mathematics in society (Gibbs, 2019) 
Table 1 MESH structural framework

Activity/goal

1. Technological activity: make sense of modeling process (constructing)

1.1. Evaluates student perception of the real situation by engaging in self-reflection

1.2. Evaluates student initial perceptions, value judgments regarding the real situation within the context of the cooperative group (Reflection)

1.3. Identify student's initial stance on the real situation (Reaction)

1.4. Utilizes reflection journals, guided reflection questions

2. Mathematical activity: examine the criteria, assumptions, and/or values embedded in productions of the modeling process (identifying, evaluating, comparing, and analyzing)

2.1. Identifies criteria, assumptions, and values used in model construction (Reflection and Self-Reflection)

2.2. Compares/contrast various models, student-produced or professional, of the real situation (Reflection)

2.3. Identify student's intermediate stance on real situation resulting from examining criteria, assumptions, and/or values embedded in productions of the modeling process (Reaction)

2.4. Utilizes reflection journals, guided reflection questions, expert, and student models of the real situation

3. Reflection activity: scrutinize the consequences of productions of the modeling process (interpret, reflect, and react)

3.1. Evaluates explicitly or implicitly stated values, assumptions, and criteria (Reflection)

3.2. Comparison of student's values to the explicitly or implicitly stated values in the model (Reflection and Self-Reflection)

3.3. Evaluates student perception of the impact of the model on their personal lives, community, or society (Reflection and Self-Reflection)

3.4. Evaluates student perception of the consequences (short and long term) of the inherent value judgments and assumptions of the model production on society (Reflection and Self-Reflection)

3.5. Evaluates the student's final stance towards the real situation after scrutinizing the consequences (Reaction)

3.6. Utilizes reflection journal, guided reflection questions

The technological activity is focused on model construction or the first three steps in Fig. 1. During the technological activity, students are engaging in the five process standards of NCTM (1989) and the three processes of OECD (2013). The mathematical activity explicitly uses traditional mathematics concepts, procedures, and formulas to analyze the model and thus engages steps four and five of Fig. 1. Additionally, the instructor presents information on the social, economic, and/or political context of the social issue. The reflection activity goes beyond the modeling process in Fig. 1 and prescribes reflections on the modeling process, models, and consequences of those models. What distinguishes the MESH tool from the traditional modeling cycle is the focus on reflection and critique of mathematics.

A pilot study using the MESH tool was conducted to ascertain if the tool stimulated reflexive discussions. Five adult participants (including three mathematics instructors) were invited to participate in a 3-h modeling workshop using the MESH tool. Results of the workshop indicated that the MESH tool did produce reflections, but the discussions were dependent on the activity. The technological activity produced the most discussions while the mathematical discussions produced very few. The MESH tool was revised based on feedback from the pilot study. The next step in the research design was to understand how the MESH tool produced reflexive discussions. Since the research on the production of reflexive discussions during mathematical modeling is sparse and we did not have a 
general idea of how the MESH tool worked, we wanted to be open to what the research would reveal. This led to a constructivist grounded theory (CGT) approach.

Research question.

The guiding question for this study is:

How can the MESH tool stimulate reflexive discussions about the role of mathematics in society during and after the technological, mathematical, and reflection activities?

\section{Methods}

This study used CGT (Charmaz, 2014) to collect and analyze data. To answer the research question, participants engaged in a 4-week modeling project using the activities of the MESH tool (see Online Resource 1 for activity details).

\subsection{Modeling task and MESH tool}

The curricular task investigated the state's minimum wage using the MESH tool. In the technological activity, students worked in cooperative groups to construct a simple model of the minimum wage based on a basic needs budget and decide whether the minimum wage should be raised to $\$ 15$. The procedures for the work on the modeling task are presented in Table 2.

In the mathematical activity, students used minimum wage data from 2005 to 2015 for the state to determine the best fit function and use the function to predict the time it would take the minimum wage to reach $\$ 15$. Additionally, there was an instructor-led discussion that provided the historical, political, economic, and social dimensions of the minimum wage including the 2004 ballot that created the minimum wage law. Finally, in the reflection activity, students worked cooperatively answering several questions about their models, the minimum wage, and the role of mathematics in society. Students completed two written reflections after each cooperative activity. The unprompted reflection was completed in class right after the activity and asked, "What are your thoughts on the activity." These were shorter than the prompted reflections which were completed outside of class and customized for each activity.

The open-ended research question reflects the researchers' desire to limit preconceptions about students' actions with the modeling task. This means that beyond the production of reflexive discussions, the researchers' opted to look to the data to understand student behavior with the modeling task. One key aspect of the modeling task was the need for students to define what constitutes basic needs. The possibility existed for a variety of basic needs models which provided an opportunity for students to see how model assumptions, criteria, and values generate different models. When these models are compared to the state or living wage models, critical reflection is possible since the state does not disclose the basis for their model. Prescribing reflections with the reflection activity and written reflection provides opportunities for contemplation of the role of mathematics in society. Since the study assumes the students have limited or no experience with mathematical modeling, the task was chosen by the instructor using Barbosa's (2003) classification of responsibility in the modeling process. 


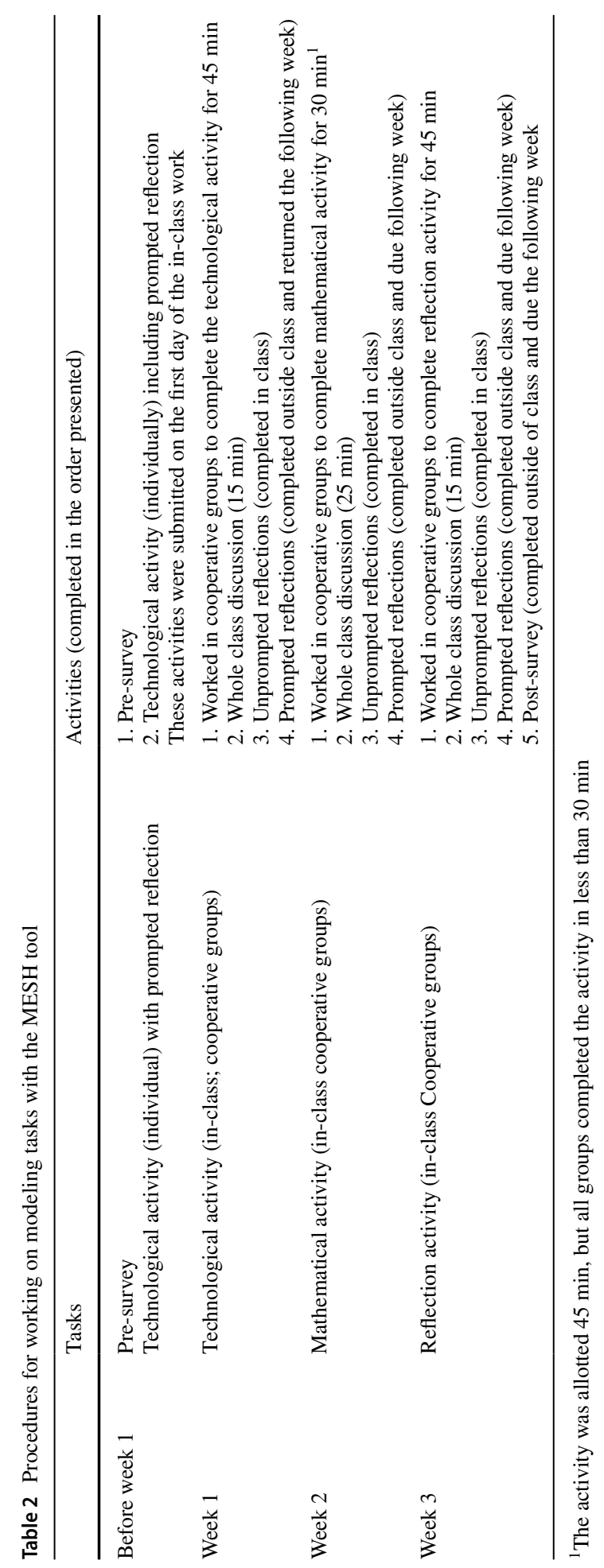




\subsection{Participants and setting}

Participants included twenty-seven students enrolled in two college algebra courses for one semester in Spring 2017 taught by the researcher at a community college in a large metropolitan area in the southernmost state. Most students enrolled at the college are eligible for a US federal education grant awarded to students from low socio-economic backgrounds, thus classifying them as economically disadvantaged populations. The local and institutional context is significant since students tend to work locally (including on-campus) in minimum wage jobs. Thus, the realistic context of the modeling task is one in which nearly all students could relate directly or indirectly. Additionally, all students involved in the study were of voting age and could participate in future elections on this legislative issue. While the courses were delivered in a blended format, all group discussions for modeling tasks took place in a face-to-face class. Participation in the study was voluntary; data were collected and utilized only for those students who gave their written consent to be a part of the study.

\subsection{Data collection and analysis}

Data collected included 810 min of audio-recorded discussions from three cooperative group activities, 180 individual reflection prompts written after each activity, and written responses from pre- and post-surveys. Students' work during the class project was treated as an archival data set for the purpose of data analysis and followed an iterative sampling plan. CGT (Charmaz, 2014) methods were used to analyze the triangulated data. Following the methodology, data analysis included coding (initial, focused, and theoretical) and making constant comparisons to develop and identify a substantive theory for stimulating reflexive discussions grounded in the empirical data.

All individual reflections were coded line-by-line by activity and then by class. When coding the cooperative group activities, it made more sense to code several lines of text together as the unit of analysis. Initial coding generated hundreds of codes. Focused coding began by coding the initial codes for each activity which led to categories. Each activity was given an overall code (see Table 5). Throughout the coding process, memos were written about the most telling codes, questions, insights, and general direction of the research. The memos, along with diagramming, created an audit trail or built-in checks and balances of the grounded theory method (Corbin \& Strauss, 2015). These memos were later sorted and organized and used in writing up the final analysis.

\subsubsection{Credibility in grounded theory studies}

Adhering to the rigorous methods of grounded theory ensures that constructed categories and concepts are grounded in the data and are generally sufficient for the credibility of the research (Charmaz, 2014). However, we address the additional criteria of resonance. The issue of resonance has to do with whether the substantive theory resonates with those with experience in the same area (Charmaz, 2014; Hadley, 2017). The four resulting concepts were reviewed by a grounded theory expert who recommended checking the concepts with professionals in the area of study. An assessment was constructed and presented a definition of each concept along with supporting excerpts from the data and a rating scale from 1 to 5. One implies the excerpt does not resonate with the definition and five indicates strong resonance. The assessments were completed by the grounded theory expert and three 
mathematics instructors who rated four of the concepts four or above indicating strong resonance. The concept of voicing mathematics was rated two by the grounded theory expert. This concept was revisited, and the definition refined. The resulting overarching main concern should be interpreted within the appropriate context, and no attempt has been made to generalize findings.

\section{Results}

First, we report students' actions during the modeling activities, how these actions led to reflexive discussions, and the resulting concepts from the coding of students' actions. Second, we formally define the overarching concept of unboxing mathematics, which is the main finding of this research. All student names represent fictitious names. Groups are labeled using the format class day (Monday or Wednesday), $\mathrm{G}$ for group, and group number $(1,2$, etc.).

\subsection{Student actions during modeling activities}

\subsubsection{The technological activity and the concept of personalizing mathematics}

The goal of the technological activity was to facilitate students' reflections on the minimum wage and the modeling process. The mathematical question was partially framed for students, but students needed to define what constitutes basic needs. To achieve this goal, students relied heavily on their collective background experience and engaged in the first five steps of the modeling process (see Fig. 1). The resulting models of the minimum wage are presented in Table 3.

Students approached the task in an ad-hoc fashion and their simplifying assumptions about what constitutes basic needs led to significant variations in the models. Some groups assumed a $\$ 15$ or $\$ 8.10$ minimum wage and proceeded to construct a budget, while others created a budget first then calculated the required minimum wage. All groups relied heavily on their collective background experiences with the problem situation. All groups included

Table 3 Summary of technological activity reports by class groups

\begin{tabular}{llllll}
\hline Groups & Minimum wage (R) & Minimum wage (C) & $\begin{array}{l}\text { Basic needs } \\
\text { budget }(\mathrm{M})\end{array}$ & Criteria & Values \\
\hline MG1 & $\$ 16.00$ & $\$ 17.44$ & $\$ 2970$ & $1 \mathrm{~A} / \mathrm{E} / \mathrm{FT}(160)$ & Did not state \\
MG3 & $\$ 30.95$ & $\$ 13.23$ & $\$ 3175$ & $1 \mathrm{~A} / \mathrm{E} / \mathrm{PT}(120)$ & Health/vacations \\
MG4 & $\$ 6.87$ & $\$ 7.80$ & $\$ 2185$ & $2 \mathrm{~A} / \mathrm{E} / \mathrm{FT}(280)$ & Did not state \\
MG5 & $\$ 9.07$ & $\$ 9.07$ & $\$ 2540$ & $2 \mathrm{~A} / \mathrm{E} / \mathrm{FT}(280)$ & Health \\
WG1 & $\$ 15.00$ & $\$ 8.77$ & $\$ 1684$ & $2 \mathrm{~A} / \mathrm{E} / \mathrm{FT}(192)$ & Family/fun \\
WG2 & $\$ 15.00$ & $\$ 14.33$ & $\$ 2260$ & $1 \mathrm{~A} / \mathrm{E} / \mathrm{FT}(160)$ & Did not state \\
WG3 & $\$ 8.20$ & $\$ 9.53$ & $\$ 1525$ & $1 \mathrm{~A} / \mathrm{E} / \mathrm{FT}(160)$ & Education/frugality \\
WG4 & $\$ 15.34$ & $\$ 15.34$ & $\$ 2455$ & $1 \mathrm{~A} / \mathrm{E} / \mathrm{FT}(160)$ & Did not state \\
\hline
\end{tabular}

$M G I$ Monday class, group 1 etc. WG1 Wednesday class group 1 etc. $R$ reported minimum wage. $C$ calculated minimum wage based on basic needs budget divided by hours per month. $M$ monthly. $A$ adult. $E$ employed. FT full time. DNS did not state. Criteria number in parenthesis refers to total hours available for work per month 
rent or mortgage, utilities (electricity, water, gas), and food as necessities. While students argued the necessity of cellular phones and cable/Internet for survival, there was little controversy about the importance of health insurance or savings. Embedded in each model are values like vacations twice per year (MG3) with a minimum wage of $\$ 30.95$ or frugality (WG3) with a minimum wage of $\$ 8.20$. MG4 reported a minimum wage (\$6.87) below the prevailing state's minimum wage of $\$ 8.10$. MG4's model highlights some mathematical errors in students' work.

The technological activity extended the modeling process to include written reflections after the activity. Table 4 presents a sample of prompted and unprompted reflections from both classes. Table 4 demonstrates the importance of prescribing reflections after modeling activities. Undoubtedly, students can reflect on their own learning, and the instructor has the opportunity to record and gauge students' thinking. The prompted reflections were completed outside of class as opposed to the unprompted reflections and are thus significantly longer. For the section of reflection on mathematics, students needed to use words instead of numbers and redefine notions like "value" and the "right answer." The written reflection gives insights into how these subtle changes in the classroom culture impacted students' understanding. Groups struggled to complete the activity in the allotted $45 \mathrm{~min}$, and concepts like model assumptions, criteria, and value proved challenging to articulate. Overall, the technological activity was the most engaging, and students' actions produced models and reflexive discussions.

Table 5 presents sample initial and focus codes that led to each category during the analysis process. The purpose of presenting some coding details is to give interested readers a sense of how the coding process developed (see Gibbs (2019) for a full methodological discussion). Initially, each of the MESH activities was given an overall category from the coding process but coding in grounded theory is tentative (Charmaz, 2016). More analytic interpretations of the categories emerged after diagramming, reviewing memos, theoretical sampling, and making constant comparisons between class, groups, individuals, and activity. The final concepts for each cooperative activity are listed in the last column of Table 5.

Table 4 Excerpts from written reflections after the technological activity

\begin{tabular}{|c|c|c|}
\hline Type of reflection & Monday class & Wednesday class \\
\hline Unprompted reflection & $\begin{array}{l}\text { I liked the activity because it gave us } \\
\text { a sense of real-life and something } \\
\text { that honestly concerns us. It made } \\
\text { us aware and shows us that math is a } \\
\text { great deal in society. (Abigail, MG3) }\end{array}$ & $\begin{array}{l}\text { This activity encourages critical } \\
\text { thought and productive debate. } \\
\text { I feel it covers an important } \\
\text { topic that needs to be discussed } \\
\text { because it affects many people. } \\
\text { Also, anything is better than func- } \\
\text { tions. (Ricky Bobbie, WG2) }\end{array}$ \\
\hline Prompted reflection & $\begin{array}{l}\text { Based on my understanding I strongly } \\
\text { agree that minimum wage should be } \\
\$ 15 \text {. There is no way people could } \\
\text { live off } \$ 8 \text { an hour, at least not in this } \\
\text { lifetime. } \$ 15 \text { an hour would at least be } \\
\text { a good start for most people. (Sashkia, } \\
\text { MG1) }\end{array}$ & $\begin{array}{l}\text { From my personal budget, I realize } \\
\text { that I did not factor in enough } \\
\text { variables such as not enough } \\
\text { money for food and water. With } \\
\text { the group the items were more } \\
\text { realistic and averaged a little } \\
\text { over } \$ 15 \ldots \text {...even if the minimum } \\
\text { wage increases to } \$ 15 \text {, so will the } \\
\text { prices of rent, gas and every other } \\
\text { item. (Molly, WG4) }\end{array}$ \\
\hline
\end{tabular}




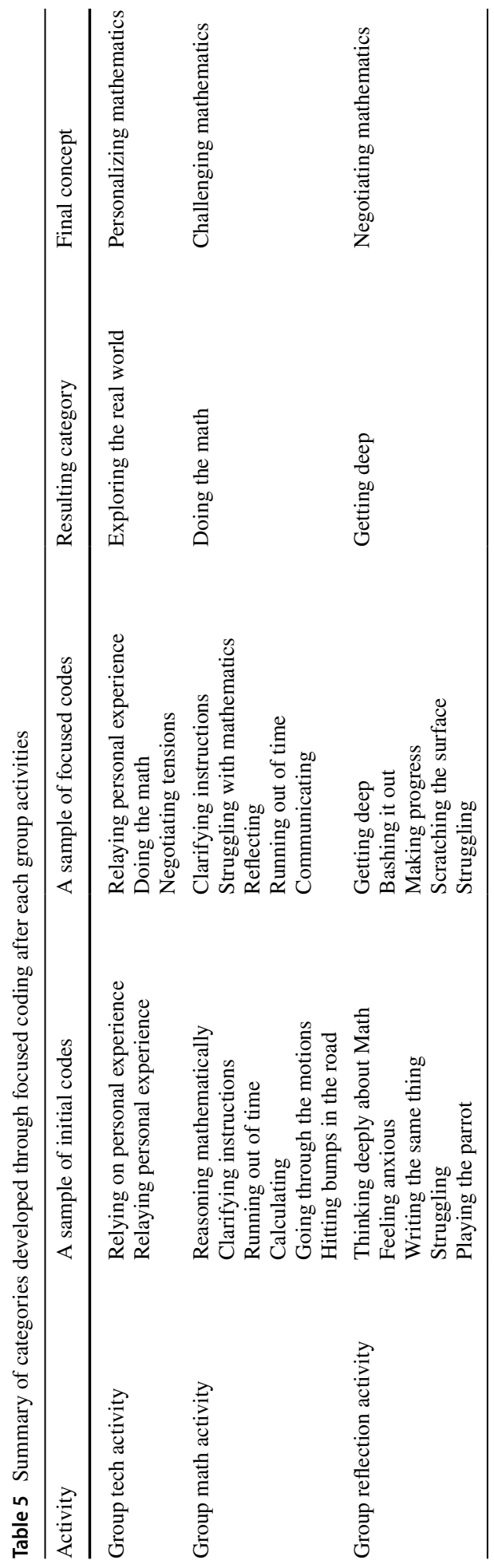


Codifying students' actions during the technology activity resulted in a process conceptualized as personalizing mathematics and is defined as referencing personal experiences with the social issue and mathematics, using personal strategies for doing mathematics, and creating new personal learning experiences during modeling activities. We illustrate how the concept of personalizing mathematics produces reflexive discussions by telling excerpts from the cooperative discussion, prompted, and unprompted reflections. Marley (MG4) shares how she used personal experience (italicized) to inform her individual budget of basic needs.

Marley: So, for me single mother 1 child [sic]working at McDonalds...total for the month that's $\$ 2,074$. And based on the budget... \$15 an hour could work for my person and 40 hours per week because, you know, that's full-time...

Abigail: Yeah. So, we can talk about them. Okay, yours, yours [sic] sounded pretty real!

Marley: Yeah. I used kind of some of my mom's figures like when we used to live in 1- bedroom apartment it was 825, and that was close.

Students' personal experiences with the social issue were welcomed and made legitimate contributions to interpreting the problem situation as well as determining model assumptions and criteria, which well-facilitated reflexive discussions. In the excerpt that follows, Marley initiates a reflexive discussion about the minimum wage after presenting her budget of basic needs.

Marley: If 2 people are making $\$ 15$ an hour, it is enough to live. [Pausing] But, I always wonder like you know how people get certificates and make $\$ 15$ an hour? So, if we raise minimum wage like, what about even when [sic] they get certificates they have to earn more than $\$ 15$ an hour?

Abigail: Because if they raise the minimum, then they have to raise the prices for everything else because it cost the workers. And then on top of that the minimum [wage] at first it was for the government so that they don't overwork the employees and pay them crumbs. So, it was only to protect their rights not just sustain a family of 3 children...if you want to sustain a family then you move up and become a manager, you get a better job, something else. You can't sustain a family at minimum wage, that can never happen.

Abigail and Marley are comparing the current minimum wage with a $\$ 15$ minimum wage and reflecting on the consequences of the different models. Informing the modeling process with personally accessible information created personal awareness, relevance, and personal expertise which produced spontaneous and prolific reflexive discussions.

Not all students had personally accessible experience with the minimum wage or budgeting. Unlike Marley, Jisoo (MG3) had no experience with budgeting or the social issue. Faced with the challenge of budgeting, her personal strategy was to omit the individual portion of the technological activity. She explained in class that she did not understand what to do and wrote the following in her unprompted reflection:

It [cooperative technological activity] showed me how expensive it is to live and how different situations affect how you live. Budgeting was difficult because I've never had to do anything like it; I had no clue how much things costs.

Jisoo's individual struggles with the model building were rooted in her lack of personal experience with the problem context, but the cooperative group became a place 
where Jisoo could learn about the minimum wage and budgeting from her peers who had first-hand experience. Each cooperative group became a personal learning space where new personal experiences were created, and reflexive discussions were present.

\subsubsection{The mathematical activity and the concept of challenging mathematics}

The goal of the mathematical activity was to reflect on the historical, political, and economic context of the minimum wage. This activity involved familiar mathematics: graphing, evaluating functions, and solving equations. Students worked systematically and finished the activity within 15-20 min. Table 6 summarizes the results of the cooperative portion of the mathematical activity. Most groups chose the linear model as the best fit for the data with a predicted $\$ 15$ minimum in approximately 45 years. Groups proceeded by moving from question to question once they found a numerical answer. WG2 was not successful in working together as evidenced by three different reported results. In the presence of familiar mathematics, reflexive discussions did not occur as expected.

Although the cooperative mathematical activity did not produce many reflexive discussions, students' written reflections after the activity provided some valuable insight into students' thinking. Table 7 presents sample excerpts from written reflections. The instructor-led discussions provided context for the minimum wage and a necessary scaffold in the reflection process. One consistent theme in students' reflections after the mathematical activity is how the new information opened their eyes which led to the in vivo code, eyeopening. During the technological activity, many students believed the minimum wage was not meant to be a living wage. Students expressed outrage after learning that the state's minimum was intended to be a living wage and was established below the county's living wage at the time.

At the end of the mathematical activity students needed to reassess their models and personal views formed during the technological activity. The code eye-opening suggests, at a minimum, that students were making these reassessments.

The relationship between students' actions and reflexive discussions during the mathematical activity is conceptualized as challenging mathematics. Sample codes leading to this concept are provided in Table 5. Challenging mathematics can be described as the process of testing students' experiences with and expectations of mathematics against social

Table 6 Summary of group results from the mathematical activity

\begin{tabular}{|c|c|c|c|c|c|}
\hline \multicolumn{2}{|l|}{ Group } & RMW & Best model & PMW & Y 215 \\
\hline \multicolumn{2}{|l|}{ MG1 } & 16 & Linear & 8.195 & DNR \\
\hline \multicolumn{2}{|l|}{ MG3 } & 30.95 & Exponential & 8.41 & 30 \\
\hline \multicolumn{2}{|l|}{ MG4 } & 6.87 & Linear & 8.195 & 45 \\
\hline \multicolumn{2}{|l|}{ MG5 } & 9.07 & Exponential & 8.41 & 30 \\
\hline \multicolumn{2}{|l|}{ WG1 } & 15.00 & Linear & 8.41 & 44.7 \\
\hline \multirow[t]{3}{*}{ WG2 } & Ricky & 15.00 & Exponential & 8.25 & 35 \\
\hline & Bankrolls & & Linear & 8.20 & 35 \\
\hline & Skai & & Linear & 8.20 & 44.7 \\
\hline \multicolumn{2}{|l|}{ WG3 } & 8.20 & Linear & 8.195 & 44.6 \\
\hline \multicolumn{2}{|l|}{ WG4 } & 15.34 & Linear & 8.195 & 44.72 \\
\hline
\end{tabular}

$R M W$ reported minimum wage $(\$ / \mathrm{Hr}$ ); $P M W$ predicted minimum wage in 2015 (\$/Hr.); Y215 years until the minimum wage reaches $\$ 15$ using the best model; DNR did not report 
Table 7 Excerpts from written reflections after the mathematical activity

\begin{tabular}{|c|c|c|}
\hline Class & Unprompted reflection & Prompted reflection \\
\hline Monday & $\begin{array}{l}\text { I think that this was a positive learning activ- } \\
\text { ity. A lot of young people in the class have } \\
\text { not experienced living on minimum wage. } \\
\text { It is a struggle to support yourself let alone } \\
\text { children. I was unaware that the state did } \\
\text { not have a minimum wage until 2004. This } \\
\text { is an eye-opener for most of us and I am } \\
\text { glad we are doing this project. (Annabelle, } \\
\text { MG5) }\end{array}$ & $\begin{array}{l}\text { The models differed because of the } \\
\text { sizes of the different families. It } \\
\text { is hard for one person living on } \\
\text { the minimum wage to do that let } \\
\text { alone a family of two or more...I } \\
\text { think that raising the minimum } \\
\text { wage to counter the increase in } \\
\text { the cost of living is fair. Maybe } \\
\text { not to } \$ 15 \text { per hour but at least to } \\
\$ 12 . \text { I believe everyone deserves } \\
\text { to earn a living wage because } \\
\text { minimum wage in our economy } \\
\text { means living in poverty. (Anna- } \\
\text { belle, MG5) }\end{array}$ \\
\hline Wednesday & $\begin{array}{l}\text { This activity was unrealistic. The food indus- } \\
\text { try is running this country. I might miss my } \\
\text { bus. (Homeslice, WG3) } \\
\text { This was an eye-opening activity. (Tony } \\
\text { Shark, WG3) }\end{array}$ & $\begin{array}{l}\text { As the group project progressed, } \\
\text { the stability of the minimum } \\
\text { wage is apparently not designed } \\
\text { to higher responsibilities. In } \\
\text { order to support basic respon- } \\
\text { sibilities, the minimum wage } \\
\text { must be raised to } \$ 15 \text { per hour. } \\
\text { The problem with raising it that } \\
\text { high is that business will not } \\
\text { hire as they would under } \$ 8 \text {. The } \\
\text { end result of our research is that } \\
\text { the minimum wage should not } \\
\text { be change higher. A couple of } \\
\text { additional cents is acceptable. } \\
\text { (Homeslice, WG3) }\end{array}$ \\
\hline
\end{tabular}

reality and making reassessments. The process of challenging mathematics can be understood by examining the initial codes going through the motions and hitting bumps in the road.

The mathematical activity used familiar mathematics (equations, graphs, formulas); thus students were doing mathematics as usual which was coded as going through the motions. However, students needed to apply mathematics in an unfamiliar context which created problems. Problematic instances were coded as "hitting bumps in the road." The following excerpt from WG1 picks up as they attempt to use their results to predict when the minimum wage will reach $\$ 15$.

Curlyfry: Predict the minimum wage. Did you get the answer or no?

Lloyd: Alright, what? For all this?

Curlyfry: Okay. Negative, it can't be a negative number though.

Lloyd: No. Wait, what am I doing?

Curlyfry: 51, that's going to be a minimum wage. I got 51.99. Oh my gosh, I was using the quadratic formula, but it gave what $t$ was. I did it wrong, okay. Let $t$ be 10 to do that.

Lloyd: The exponential growth.

Curlyfry: \$7.6, you didn't get that?

Lloyd: Okay, you're doing the linear and the quadratic right now. 
Curlyfry: I'm just plugging it into the equation. I guess the quadratic then.

Lloyd: Yeah, that's what I did, but that's not what I got.

Curlyfry: You didn't get quadratic.

Lloyd: No. Shoot, stop.

WG1 chose the linear model as the best fit for the data, yet they proceeded to do some calculations with the quadratic and exponential functions. When Curlyfry says, "Okay. Negative, it can't be a negative number though," she hit a bump in the road. Bumps in the road could represent problems interpreting modeling results, working with mathematics concepts, or working cooperatively and affected all groups. These problems are inconsistent with their past mathematics experience and are not resolved by merely re-calculating figures. In this activity, students' mathematics experience and expectations were challenged in various ways. At the same time, the instructor-led discussion challenged their perceptions.

Opportunities for reflexive discussions existed; however, most groups engaged in mathematical discussions (Barbosa, 2006). The following excerpt from WG4 provides an example.

Kaiti: According to the best model how much longer until the actual minimum wage has reached 15 ? Then you got to solve for that. It will take at least 44 years. Elijah: Okay, 44 years.

Kaiti: Yeah. At least 44 years. Round it up to 45 years. All right. Did you get the same answer?

Elijah: I got 45 . It comes out to 4544 , yeah, between there.

Kaiti: This is according to the linear model.

Elijah: 45 years.

Molly: What is the formula that you used?

The italicized text represents opportunities where students could have engaged in reflexive discussions. Molly's question about the formula moves the focus to mathematical concepts.

The empirical data suggest a dual nature to the notion of challenge relative to the presence of reflexive discussions. Underlying structural challenges hindered reflexive discussions during the cooperative activity. This result supports Barbosa's (2009) explanation that reflexive discussions must click for students, and they cannot be forced to produce them.

At the same time, the revelation of novel information about the social issue and the probing questions during the instructor-led discussions did stimulate reflexive discussions by challenging students' perceptions. Scaffolding by the instructor in this instance is used to "draw out" internal considerations about the models.

\subsubsection{The reflection activity and the concept of negotiating mathematics}

The reflection activity extends the modeling cycle and is not mathematical in nature. The activity used a series of questions to help students think about the consequences of the models and the role of mathematics in society. Students struggled with the question as illustrated by WG4 in the following excerpt.

Elijah: I don't understand what's direct and indirect.

Kaiti: Direct would be mostly like what are direct benefits or negative aspects of it. I guess the direct stuff would be like stuff that you can see immediately from when you 
make a model. If the model is good or is bad and it does this or that. Indirect would be like well because we're looking at like this it could affect in other ways.

Kaiti's response is typical of how students relied on their peers, the instructor, or Google for clarification. The ambiguity and variations in the answers were also problematic for many students as they are accustomed to seeking and getting one "right" answer.

Table 8 presents sample responses to the final question about the role of mathematics in determining the minimum wage. Students could readily connect the use of mathematics as a practical tool to the minimum wage models. It was more challenging for students to articulate the connections between their minimum wage models and citizenship and democracy. Ricky Bobby made a direct association to the impact on immigration. Students were less likely to connect the minimum wage to indirect impacts on citizens and democracy. The reflection activity of the MESH tool prescribed reflections. Codes relating students' actions during the reflection activity and the presence of reflexive discussions were conceptualized as negotiating mathematics (see Table 5 for sample codes). Negotiating mathematics is defined as the constant balancing of experiences with and expectations of mathematics during mathematical modeling activities. The search for the right answer during the reflection activity is a key element of the process of negotiating mathematics. The right answer is a part of their mathematics experience, but the answers varied during the activities and required constant balancing to make progress.

Iconic and Jisoo (MG3) are trying to differentiate between material and immaterial technology.

Iconic: So, I will say cultural practices because when I think material like I feel something more like materialistic, would that be okay, cultural practices?

Jisoo: For labor savoring?

Iconic: Yes.

Jisoo: Yes. So, we can at least have an answer.

Later in the conversation, Jisoo expresses great pride in their work.

Jisoo: I'm proud of us we have an answer for one of them. It's progress.

Iconic: Okay. Cultural practices are [sic] the role of labor savoring [sic] technology.

Jisoo: Okay, the next terrifying question is how does your model of minimum wage impacts social development for prioritizing human need especially the poor and developing society?

Searching for one right answer in mathematics class may be associated with sociomathematical norms (Cobb \& Yackel, 1996; Yackel \& Cobb, 1996) created within typical traditional mathematics classroom communities and classroom absolutism (Skovsmose, 1998), the structuring of classroom communication around the assumption that there's only one right answer. On one level, the reflection activity was a tacit renegotiation of those norms and assumptions which proved to be problematic.

The search for the right answer often led to students getting deep, an in vivo code. After answering the first several questions of the reflection activity, MG4 was overtaken by silence. After $1 \mathrm{~min}$ of complete silence in the group, they proceeded.

Toby: How does the minimum wage impact?

Marley: How does? [reading quietly]

Toby: How does the minimum wage impacts citizenship and democracy?

Alana: Mm? 


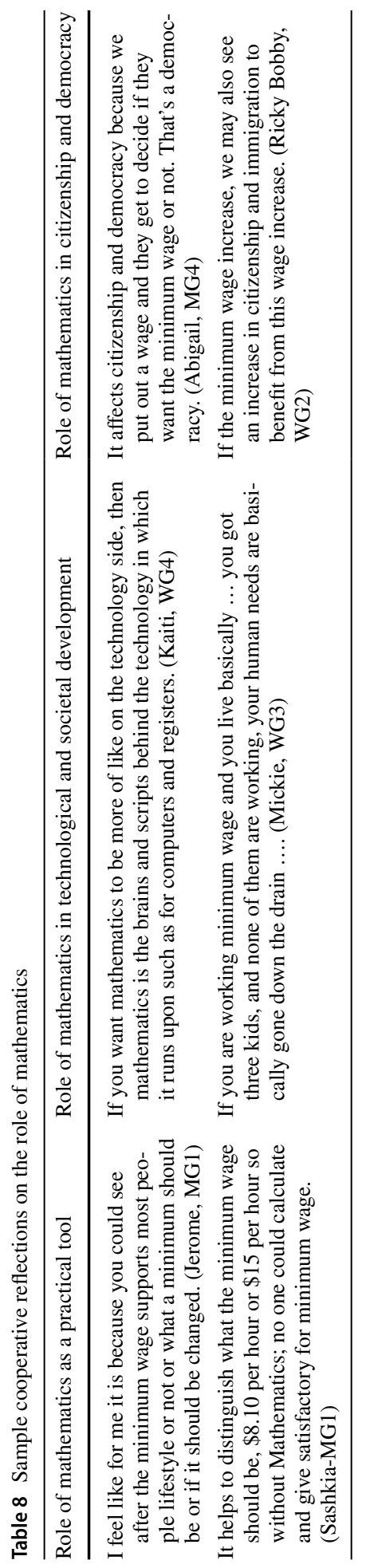


Toby: How does it impact citizenship and democracy? How does it impact citizen-

ship and democracy?

Marley: This is...this is getting too deep.

Marley's utterance captures students' recognition of the need for critical thinking uncharacteristic of mathematics in school. Students struggled to connect mathematics in school with mathematics in real life.

While students did engage in reflexive discussions, the process was difficult. The reflection activity challenged students' thinking and students did not resolve these problems in 75 min. Instead, they progressed as best they could towards the goal of succeeding on the assignment. Students needed to renegotiate individual and collective mathematical beliefs and values about mathematics in school and mathematics in the real world. It is in this sense that the word negotiating is understood.

\subsubsection{The concept of voicing mathematics: Giving thought to modeling activities in and outside the school mathematics box}

Central to the aforementioned processes is a basic social process conceptualized as voicing mathematics. The concept of voicing mathematics describes how students reflect (communicate and express feelings, attitudes, and positions about mathematics and the social issue) during modeling activities using the MESH tool. Table 9 provides a list of sample codes that led to the concept of voicing mathematics. In the traditional teacher-centered classroom, opportunities for reflection are novel for most students. Students' reflections were present during cooperative activities and written reflections after each activity. Students' reflections can be described as collegial during cooperative discussions, emotional in response to unprompted reflections, and rational in prompted reflections.

Table 10 reports the prompted reflections of Alana (MG4) across the entire project with the MESH tool and exemplifies the properties of voicing mathematics. Alana's position is relatively unchanged, but her perspective about the social issue is expanded after each activity.

Her prompted reflections show how she interpreted and internalized the different and new information she learned during the cooperative activities. The cooperative discussions had the effect of augmenting Alana's position. She is in favor of increasing the minimum wage but not to $\$ 15$ in the end which is a change from her initial position.

Ultimately, students could take up action outside of class in response to reflecting on the role of mathematics, but this property of voicing mathematics was not directly observed. Alana's reflections demonstrate how these cumulative communicative actions led to an adaptive characteristic of students' reflections. Adaptive does not mean students adopted any point of view but instead refers to the progressive development of their critical competence from the social interaction and written reflections.

Stimulating reflexive discussions about the role of mathematics in society during modeling activities depended on giving students a "voice." Certainly, students' discourses during mathematical modeling at the micro-social level are far more nuanced and require further study using discourse analysis; however, in this study, the word "voicing" and the concept of voicing mathematics accounts for verbal and non-verbal forms of reflecting as well as any silence which does not necessarily indicate the absence of reflection. In the next section, we integrate the results presented into one overarching concept that captures how the MESH tool stimulated reflexive discussions. By conceptualizing the process of stimulating 


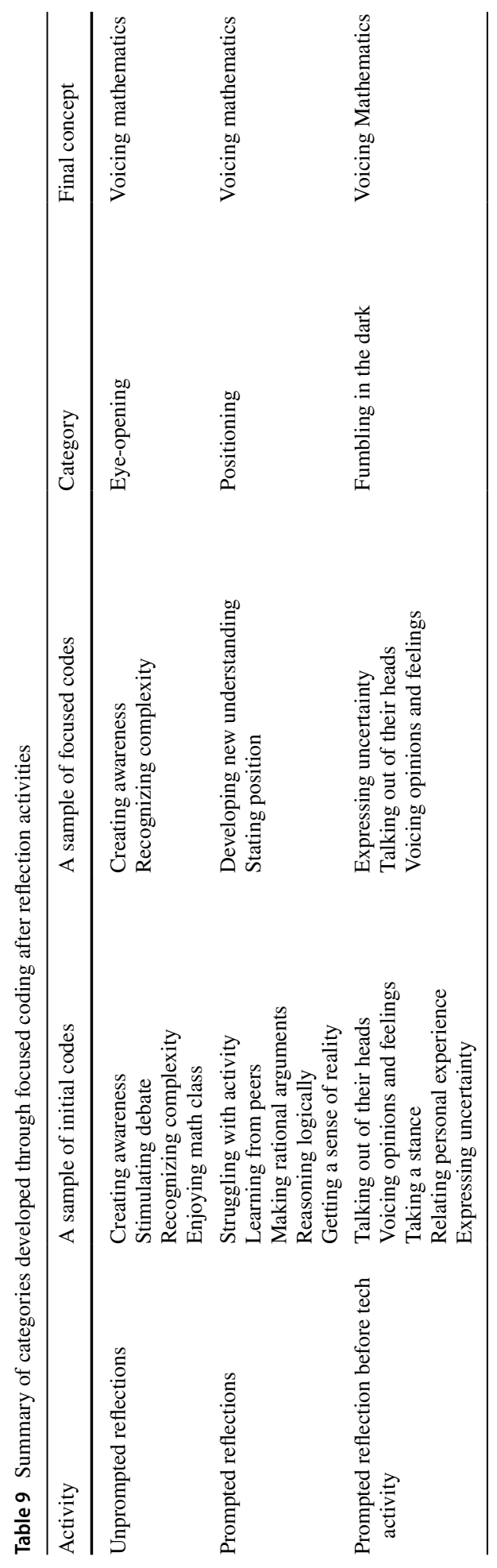


Table 10 Tracing the properties of the concept voicing mathematics during MESH activities (Alana, MG4)

Prompted reflection activity/ Alana (MG4)
property of voicing math-
ematics

Before tech (1B)

Emotional and assertive

After tech (1E)

Rational and assertive

After math (2C)

Rational and alterative

After reflection (3C)

Rational and adaptive
The minimum wage should be increased to $\$ 15 \ldots$ if the minimum wage should increase, they would not have to work so many jobs and they would have more time with their kids. The companies can afford to pay each employee $\$ 15$ an hour when their CEOs and owners are making millions.

Increasing the minimum wage would force businesses to lay off employees because not every company is a Walmart or McDonalds...The assumption that if the minimum wage is raised the price of consumer goods would also increase was brought up during our group discussion and I feel that nothing will change if the minimum wage is increased and the cost of living increased with it.

I feel that minimum wage should be raised to $\$ 15$ because when the minimum wage was introduced the government expected for people to live on it...I understand that if the minimum wage increases so will the cost of living, but I feel regardless the cost of living will always go up.

If we do the math, we'll see that it's not enough...I feel the minimum wage should increase, but I do not think it should increase to $\$ 15$. We need to come... to a compromise that does not destroy the economy in the process.

reflexive discussions in this way, we dispense with the MESH tool and provide a practical framework for developing modeling activities that will help students understand the role of mathematics in society.

\subsection{The concept of unboxing mathematics: Creating a culture of modeling as critic}

Further analysis of the four concepts presented led to an overarching conceptualization of how reflexive discussions are stimulated. Before naming this concept, we briefly discuss the context in which the results should be interpreted. Two important contextual factors in this study are the MESH tool and the notion of traditional or school mathematics. Within these contexts, two important dichotomies emerged relative to students' understanding of school mathematics: mathematics in school versus mathematics in real life and being "good at math" versus being "bad at math." These shared views of the learning environment and mathematics ability made clear that stimulating reflexive discussions was as much about the interactions within the classroom environment as it was about individual perceptions and understanding of mathematics. Bauersfeld (as cited in Yackel \& Cobb, 1996) suggests that participation in mathematics classroom processes "is participating in a culture of using mathematics or better: a culture of mathematizing as practice" (p. 459). The empirical data indicate that students understand this culture of mathematizing as practice as mathematics in school and is distinct from mathematics they encounter every day. Further, being "good at math" or "bad at math" in school impacted students' personal, social, and professional engagement of mathematics in and out of school. These dichotomies, more than any other contextual elements, tacitly created the backdrop in which the MESH tool was applied.

Under the contextual factors outlined, engaging students in modeling activities with various opportunities for critique and reflection can be conceptualized as a process of 
unboxing mathematics. Figure 3 illustrates the logic of unboxing mathematics. Unboxing mathematics involves four sub-process: personalizing mathematics, challenging mathematics, negotiating mathematics, and voicing mathematics. The purpose of unboxing mathematics is to establish the relevance of mathematics in everyday life represented by the lower triangle in Fig. 3.

The relevance of mathematics to students is hierarchical. The more concrete and direct use of mathematics as a practical tool, the more explicit and relevant the role of mathematics. As the use of mathematics becomes more abstract, invisible, and indirect as in the case of mathematics used in decision-making in citizenship and democracy, the more implicit and less relevant the role of mathematics. Figure 3 illustrates the complicated

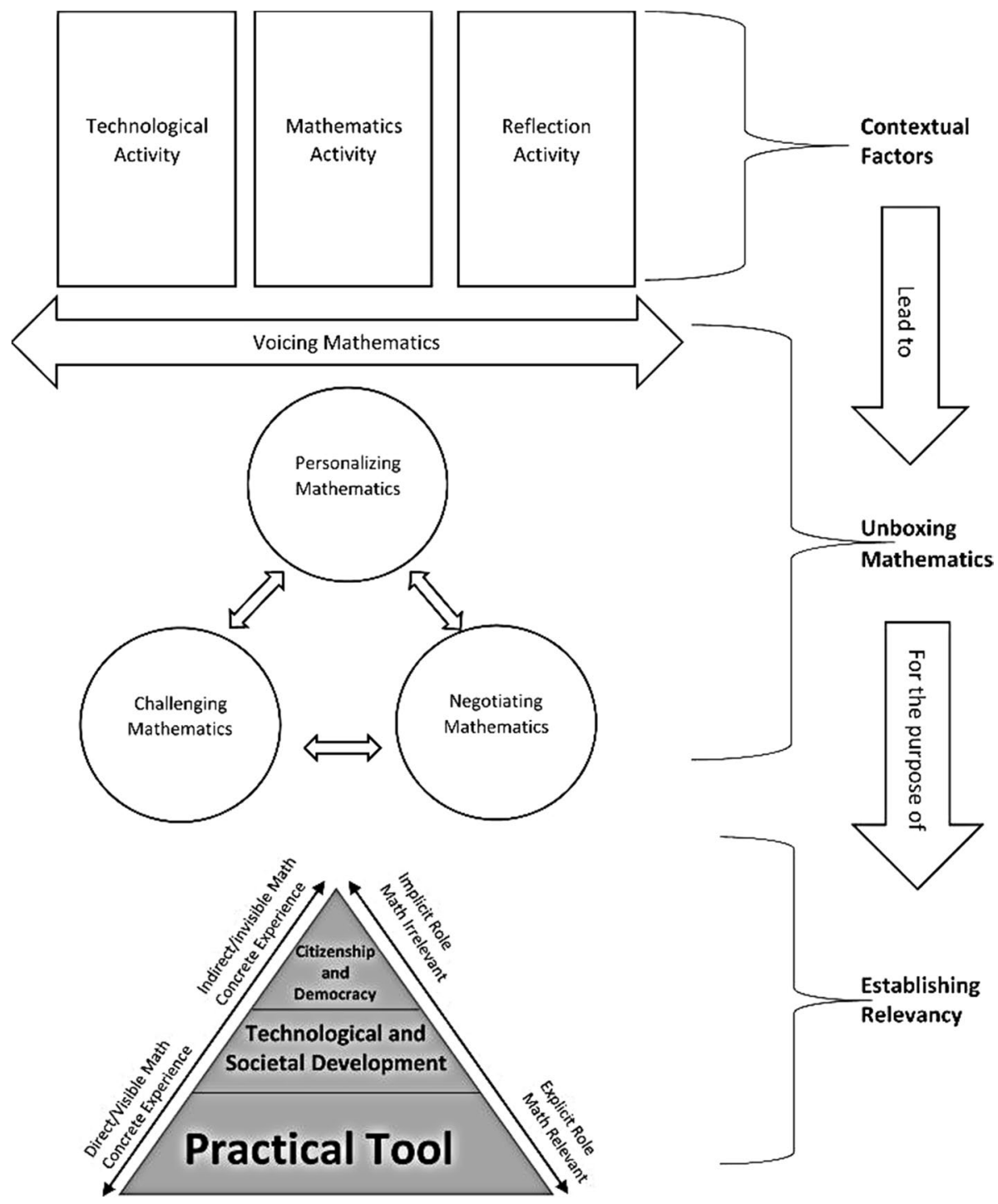

Fig. 3 The logic model of unboxing mathematics (Gibbs, 2019) 
relationship between mathematics utility and its role in everyday life. Unboxing mathematics accounts for both the communal and social development of reflexive discussions and provides a framework for creating a classroom culture of modeling as critic (Barbosa, 2006) that should generate reflexive discussions about the role of mathematics in society.

\section{Discussion and conclusion}

Motivated by the need to help students understand the role of mathematics in society and identify pedagogical practices for stimulating reflexive discussions during mathematical modeling, the tool MESH was constructed. A constructivist grounded theory approach was used to answer the following research question: How can the MESH tool stimulate reflexive discussions about the role of mathematics in society during and after the technological, mathematical, and reflection activities? Our findings indicate that the MESH tool stimulated reflexive discussions during the technological, mathematical, and reflection activities by engaging students in integrated processes of personalizing, challenging, negotiating, and voicing mathematics (see the "Results" section). Together, these processes are conceptualized as unboxing mathematics, emphasizing the need to transform the traditional mathematics classroom into a classroom culture where critique and reflection are normative.

The primary result of this study is the framework of unboxing mathematics which dispenses with the MESH tool and provides a way of organizing socio-critical modeling activities that should stimulate reflexive discussions. What would this look like in practice? First, in an ideal world, students would choose the real-world issue they wish to investigate and construct mathematical models to solve the problem (personalizing mathematics). The choice of task is usually a collaboration between teacher and students (Barbosa, 2003) and depends on the required mathematical competence. The finding that personalizing mathematics is necessary for stimulating reflexive discussions is consistent with the modeling literature on value creation (Park, 2014). Using Makiguchi's (1981-1988) value creation as a lens to understand students' mathematical disposition and value of mathematics during mathematical modeling, Park's study (2014) demonstrated that modeling tasks involving students' personal lives could spark their interest, which led students to view mathematics as a means of creating value for the benefit and society's sake. Requiring personalization during the model construction process ensures value creation during modeling by engaging students in a social issue that is very familiar personally, socially, and culturally. This in turn contributes to robust, rich, and eyeopening reflections and allows students to connect mathematics to their lives in individually meaningful ways.

Secondly, the instructor provides activities that help students analyze and validate their models as well as provide the social, economic, or political context for the real-world issue under investigation (challenging mathematics). This result highlights the importance of challenging students' experience with and expectations of mathematics and is aligned with Maass, Doorman, Junker, and Wijers (2019) study on using socio-scientific issues (SSIs) and inquiry-based learning (IBL) to teach citizenship education. Our study, like Maass et al. (2019), engages students in the social, political, and economic controversy that is of interest to them and is consistent with Stephan et al.'s (2021) findings on the necessity of contextual relevance in developing students' critical mathematics consciousness. One 
caveat is the possible imposition of teacher values. Teachers can mitigate this situation by being conscientious of personal biases and beliefs; keeping questions sufficiently open to encourage students to formulate their position; and limiting conversations to providing facts, statistics, and data without suggesting a position.

Thirdly, the instructor should prescribe reflections (negotiating mathematics) at the end of the modeling process. It is important to note that the process of negotiating mathematics occurs throughout the modeling activity as students are constantly balancing their mathematical results with reality. However, the results of this study demonstrate that prescriptive critique and reflection must extend beyond the modeling cycle and deliberately contemplate the consequences of the productions of modeling in the real world. In this study, students were not accustomed to articulating reflections about a real-world problem or writing in a traditional mathematics classroom. Thus, perceptions (students and teachers) of school mathematics classroom culture, mathematics in school, and mathematics in the real world delimit the production of reflexive discussion.

Finally, verbal and written reflections throughout are fundamental to the entire process (voicing mathematics). The final important action of the MESH tool in stimulating reflexive discussion was giving students a "voice" in a traditional class where they are usually silent recipients of knowledge. Voicing mathematics is present throughout all activities using the MESH tool, embodies psychological goals (Kaiser \& Sriraman, 2006) in modeling, and becomes the glue that holds the whole process together. The concept of voicing mathematics captures both individual and collective reflections on the learning process which stimulates and regulates students' critical reflective competence as a form of empowerment. According to Brown (2013), requiring year 6 students to reflect on their mathematics and explicate their thinking contributed to their perception of their contribution to interpreting the real-world situation and connecting mathematics to the real world. Voicing mathematics considers Barbosa's (2006) three types of discussions (technological, mathematical, and reflexive) during modeling and Skovsmose's (2012) three demands of critique (self-reflections, reflections, and reaction).

The research findings cut across the eight themes for the future of mathematics education research outlined by Bakker et al. (2021): approaches to teaching, goals of mathematics education, teacher professional development, technology, equity, diversity, and inclusion, affect, and assessment. This study highlights the contributions to the themes.

First, unboxing mathematics disrupts the traditional mathematics classroom and offers an approach to teaching that is engaging, inclusive, and empowering. The project introduced implicitly a counter-narrative to historical beliefs about mathematics values (Bishop, 1999) which Curlyfry (WG1) confirmed in protest in her written reflection, "this isn't math." The explicit and tacit renegotiation of mathematics educational values (Bishop, 1999) like working step-by-step towards one right answer was problematic for students. The notion that mathematics always provides a "right" answer has implications for how students see their world mathematically. For example, students as citizens may interpret mathematical data, models, or statistics presented during a crisis as unbiased facts and use these facts to make life altering decisions. However, as Kollosche and Meyerhöfer (2021) argued, expert knowledge is required to interpret the mathematical information presented in the media about COVID-19 that led to misinformation and manipulation. Recognitions of such limitations of mathematics education and the way mathematics operates during crisis (Skovmose, 2021) demand more approaches to teaching like unboxing mathematics.

Second, we commenced this research with the hope of bridging the gap between educational goals of school mathematics and societal goals of developing critical, engaged, 
and productive citizens. Unsurprisingly, central elements to the production of reflexive discussions were collaboration in cooperative groups and mathematical modeling. According to Yackel and Cobb (1996), interaction is critical to negotiating shared meanings and developing individual reasoning and sense-making. Formalizing an inquirybased learning approach (Artigue \& Blomhøj, 2013; Maass et al., 2019) would be one possible way of implementing the resulting framework. Relevant contingencies to implementing the framework of unboxing mathematics in practice include allotting adequate time for modeling activities, student modeling competencies, and teacher-specific knowledge.

Unboxing mathematics is not a recipe for producing reflexive discussions in practice. After all, classroom cultures in general are unique and represent unwritten rules between teacher and students. We present one possible way to reframe the traditional mathematics classroom into one that is conducive to reflection and critique. Any attempt at implementing this novel framework will be guided by the local and institutional context of the mathematics classroom.

Also, there is a recognition that stimulating reflexive discussion is an ongoing lifelong process for students as critical citizenship requires informed engagement and action (Frankenstein, 1983; Niss, 1994; Skovsmose, 1994) outside of the classroom. Now more than ever, a mathematics education necessitates action to be effective. Activism was not directly investigated in this study but would be a useful final step in any implementation of unboxing mathematics and demands further research. Our primary recommendation is a study of the framework of unboxing mathematics in various contexts.

In conclusion, findings from this research contribute to pedagogical practices in mathematical modeling and to the broader socio-political concerns of mathematics education as outlined in curriculum documents globally. Since modeling is defined as a school activity (Barbosa, 2006), the integrative framework and concepts of this research provide insights into the necessary classroom conditions and a tractable process for stimulating reflexive discussions amenable to classroom practice. Additionally, if mathematics literacy develops critical, productive, engaged citizens, the mathematics classroom must intentionally prioritize critique and reflection.

\section{Availability of data and material}

Code availability Not applicable.

Author's contributions This manuscript is based on the data from a larger study conducted by Antonnette M. Gibbs under supervision of Joo Young Park.

\section{Declarations}

Ethics approval Permissions were obtained from the appropriate ethics committees.

Consent to participate Informed consent was obtained from all participants.

Conflict of interest The authors declare no competing interests. 


\section{References}

Artigue, M., \& Blomhøj, M. (2013). Conceptualizing inquiry-based education in mathematics. ZDM-Mathematics Education, 45, 797-810. https://doi.org/10.1007/s11858-013-0506-6

Atweh, B. (2007). Pedagogy for socially response-able mathematics education. Paper presented at the annual conference of the Australian Association of Research in education. Fremantle, West Australia.

Bakker, A., Cai, J., \& Zenger, L. (2021). Future themes of mathematics education research: An international survey before and during the pandemic. Educational Studies in Mathematics, 107, 1-24. https://doi. org/10.1007/s10649-021-10049-w

Barbosa, J. C. (2003). What is mathematical modelling? In S. J. Lamon, W. A. Parker, \& S. K. Houston (Eds.), Mathematical modelling: A way of life. ICTMA11 (pp. 227-234). Horwood Publishing.

Barbosa, J. C. (2006). Mathematical modelling in classroom: A socio-critical and discursive perspective. ZDM-Mathematics Education, 38(3), 293-301.

Barbosa, J. C. (2009). Mathematical modelling, the socio-critical perspective and the reflexive discussions. In M. Blomhøj \& S. Carriera (Eds.), Mathematical applications and modelling in the teaching and learning of mathematics proceedings from the topic study group 21 at $11^{\text {th }}$ international congress on mathematics education in Monterrey, July 6-13, 2008 (pp. 133-143). Roskilde University.

Barbosa, J. C. (2010). The students' discussions in the modeling environment. In R. Lesh et al. (Eds.), Modeling students' mathematical modeling competencies (pp. 365-372). https://doi.org/10.1007/ 978-1-4419-0561-1_31

Bishop, A. J. (1999). Mathematics teaching and values education-an intersection in need of research. ZDM-Mathematics Education, 31(1), 1-4. https://doi.org/10.1007/s11858-999-0001-2

Blomhøj, M. (2009). Different perspectives in research on the teaching and learning mathematical modelling - Categorizing the TSG21 papers. In Mathematical applications and modelling in the teaching and learning of mathematics. Proceedings from the topic study group 21 at $11^{\text {th }}$ international congress on mathematics education in Monterrey, July 6-13, 2008 (pp. 1-17). Roskilde University.

Blum, W., Galbraith, P. L., Henn, H. W., \& Niss, M. (Eds.). (2006). Modelling and applications in mathematics education: The $14^{\text {th }}$ ICMI study (new ICMI study series). Springer.

Brown, Jill P. (2013). Inducting year 6 students into "A Culture of Mathematising as Practice". In G.A. Stillman et al. (Eds.), Teaching mathematical modelling: Connecting o research and practice, international perspectives on the teaching and learning of mathematical modelling (pp. 295-305). https://doi.org/10.1007/978-94-007-6540-5_24

Charmaz, K. (2014). Constructing grounded theory. Sage Publications Inc..

Cobb, P., \& Yackel, E. (1996). Constructivist, emergent, and socio-cultural perspectives in the context of developmental research. Educational Psychologist, 31(3/4), 175-190.

Corbin, J., \& Strauss, A. (2015). Basics of qualitative research: Techniques and procedures for developing grounded theory (4th ed.). Sage Publications, Inc..

D’Ambrosio, U. (1999). Literacy, matheracy, and technocracy: A trivium for today. Mathematical Thinking and Learning, 1(2), 131-153.

Frankenstein, M. (1983). Critical mathematics education: An application of Paulo Freire's epistemology. Journal of Education, 165(4), 315-339.

Galbraith, P. (2012). Models of modelling: Genres, purposes or perspectives. Journal of Mathematical Modelling and Application, 1(5), 3-16.

Jablonka, E., \& Gellert, U. (2007). Mathematisation - Demathematisation. In U. Gellert \& E. Jablonka (Eds.), Mathematisation and demathematisation: Social, philosophical, and educational ramifications (pp. 1-18). Sense Publishers.

Gibbs, A. M. (2019). Socio-critical mathematics modeling and the role of mathematics in society (unpublished doctoral dissertation). Florida Institute of Technology.

Gutstein, E. (2006). Reading and writing the world with mathematics: Toward a pedagogy of social justice. Taylor \& Francis Group.

Hadley, G. (2017). Grounded theory in applied linguistics research: A practical guide. Routledge.

Julie, C. (2002). Making relevance relevant in mathematics teacher education. In I. Vakalis, D. Hughes Hallett, D. Quinney, \& C. Kourouniotis (compilers). Proceedings of the 2nd international conference on the teaching of mathematics (ICTM-2). Wiley [CD-ROM].

Kaiser, G., \& Sriraman, B. (2006). A global survey of international perspectives on modelling in mathematics education. ZDM-Mathematics Education, 38(3), 302-310.

Kollosche, D., \& Meyerhöfer, W. (2021). COVID-19, mathematics education, and the evaluation of expert knowledge. Educational Studies in Mathematics. https://doi.org/10.1007/ s10649-021-10097-2 
Makiguchi, T. (1981-1988). Makiguchi Tsunesaburo zenshu [the complete works of Tsunesaburo Makiguchi] 10 Vols. Daisan Bunmeisha.

Maass, K., Doorman, L. M., Jonker, V. H., \& Wuers, M. M. (2019). Promoting active citizenship in mathematics teaching. ZDM-Mathematics Education, 51(6), 991-1003.

National Council of Teachers of Mathematics. Commission on Standards for School Mathematics. (1989). Curriculum and evaluation standards for school mathematics. Author.

National Governors Association Center for Best Practices, Council of Chief State School Officers. (2010). Common core state standards for mathematics. National Governors Association Center for Best Practices and the Council of Chief State School Officers.

Niss, M. (1994). Mathematics in society. In Biehler et al. (Eds.), Didactics of mathematics as a scientific discipline (pp. 367-378). Kluwer Academic Publishers.

Organization for Economic Cooperation and Development (2013). PISA 2012 assessment and analytical framework: Mathematics, reading, science, problem solving and financial literacy. Retrieved from 10/17887/9789264190511-en

Park, J. (2014). 'Value creation' through mathematical modeling: Students' mathematics dispositions and identities developed in a learning community (doctoral dissertation). Retrieved from ProQuest Dissertations \& Theses Global. (order no. 3622713). Retrieved from http://search.proquest.com. portal.lib.fit.edu/docview/1548332299?accountid=27313. Accessed Feb 2015

Pollak, H. (2007). Mathematical modelling - a conversation with Henry Pollak. In W. Blum, P. Galbraith, H. W. Henn, \& M. Niss (Eds.), Modelling and applications in mathematics education (pp. 109-120). Springer.

Seah, W. T., \& Bishop, A. J. (2002). Values, mathematics, and society: Making the connections. In C. Vale, J. Roumeliotis, \& J. Horwood (Eds.), Valuing mathematics in society (pp. 105-113). Mathematical Association of Victoria.

Skovsmose, O. (1994). Towards a critical mathematics education. Educational Studies in Mathematics, $27(1), 35-57$.

Skovsmose, O. (1998). Linking mathematics education and democracy: Citizenship, mathematical archaeology, mathemacy and deliberative interaction. ZDM-Mathematics Education, 30(6), 195-203.

Skovsmose, O. (2011). An invitation to critical mathematics education. Sense Publishers.

Skovsmose, O., \& Greer, B. (2012). Seeing the cage? The emergence of critical mathematics education. In O. Skovsmose \& B. Greer (Eds.), Opening the cage: Critique and politics of mathematics education (vol. 23). Sense Publishers.

Skovsmose, O. (2021). Mathematics and crises. Educ Studies in Mathematics. https://doi.org/10.1007/ s10649-021-10037-0

Stephan, M., Register, J., Reinke, L., et al. (2021). People use math as a weapon: Critical mathematics consciousness in the time of COVID-19. Educational Studies in Mathematics. https://doi.org/10.1007/ s10649-021-10062-Z

Yackel, E., \& Cobb, P. (1996). Sociomathematical norms, argumentation, and autonomy in mathematics. Journal for Research in Mathematics Education, 27(4), 458-477. https://doi.org/10.2307/749877

Publisher's note Springer Nature remains neutral with regard to jurisdictional claims in published maps and institutional affiliations. 